\title{
Femmes, waqf et droit de propriété en Iran à l'époque safavide
}

\author{
Mohammadreza Neyestani \\ TELEMME-MMSH, AMU \\ mrneyestani@gmail.com
}

\begin{abstract}
In Safavid society in Iran (16th-18th centuries), women played a role, albeit to a lesser degree than men, through the institution of waqf. Using this instrument of waqf, women left traces in society which indicate their rights with respect to property as well as the role which they played in the cultural, religious, and charitable sectors at that time. Women in the Safavid capital, Isfahan, invested in society according to their means by exercising their rights with regard to their property by consecrating a part of their assets towards financing charitable works of general interest via the creation of waqf.

This article studies certain aspects regarding the rights of property belonging to women during the Safavid period by analyzing primary documents from that era, with particular attention focused on waqf foundation charters which are preserved today in the archives of Isfahan.
\end{abstract}

\section{Keywords}

women - waqf - Safavid - Iran - rights of property - charity

\section{Résumé}

Dans la société safavide en Iran ( $\mathrm{du} 16^{\mathrm{ème}}$ au $18^{\text {ème }}$ siècle), les femmes jouaient un rôle, quoique à un moindre degré que les hommes, à travers les waqfs. Par ce moyen, elles ont laissé des traces qui indiquent leurs droits de propriété ainsi que le rôle qu'elles jouaient dans les domaines culturels, religieux et caritatifs à l'époque. Les femmes de la 
capitale safavide s'investissaient dans la mesure de leurs moyens et grâce à leurs droits de propriété dans ces questions en consacrant une partie de leurs biens aux œuvres d'intérêt général et caritatives, à travers le waqf.

Dans cet article, nous étudions certains aspects du droit de propriété accordé aux femmes qu'elles exerçaient à l'époque safavide en nous appuyant sur les documents d'époque, particulièrement, sur les chartes de fondation des waqf conservées dans les archives d'Ispahan.

\section{Mots clés}

waqf - femme - droit de propriété - safavide - Iran

Dans la société safavide d'Iran ( $\mathrm{du} 16^{\mathrm{e}}$ au $18^{\mathrm{e}}$ siècle), les femmes jouaient un rôle non négligeable, quoique à un moindre degré que les hommes, à travers les waqfs. Par ce moyen, elles ont laissé des traces qui indiquent leur droit de propriété ainsi que le rôle qu'elles assumaient dans les domaines culturel, religieux et caritatif de l'époque. Dans cet article, nous étudions certains aspects du droit de propriété accordés aux femmes qu'elles exerçaient à l'époque safavide en nous appuyant sur les documents d'époque, particulièrement, sur les chartes de fondation des waqfs conservées à l'Archive de l'Organisation des Waqfs et des CEuvres Caritatives d'Ispahan. Certains de ces documents qui remontent jusqu'à l'époque safavide sont des originaux mais les autres ne sont que des copies. Cela dans la mesure où les originaux se sont perdus avec le temps et les administrateurs de ces waqfs ou d'autres personnes ayant rapport à ces fondations ont déposé des copies des originaux aux archives ou, enfin, les originaux se trouvent dans les familles ou les descendants des fondateurs/ fondatrices des waqfs qui ne souhaitent pas les léguer aux archives bien qu'il soit possible que la prochaine génération le fasse.

La totalité des documents des waqfs d'Ispahan qui se trouvent actuellement à l'Archive de l'Organisation des Waqfs et des OEuvres Caritatives d'Ispahan depuis l'époque safavide jusqu'à nos jours sont au nombre de $800^{1}$. Ils sont rassemblés, relus et corrigés par des spécialistes et sont publiés par l'Organisation des Waqfs et des OEuvres Caritatives d'Ispahan dans un recueil en 12 volumes. Ce recueil intitulé Documents des waqfs d'Ispahan est la référence pour les chartes des waqfs utilisés dans cet article.

1 Ashkiwarî, 1387 / 1430 / 2009, v. l1, p. 10. 


\section{La définition et la nature du waqf}

Commençons d'abord par une introduction afin de définir les expressions utilisées dans cet article.

1) Sens littérale du waqf: se tenir debout, s'arrêter².

2) Sens juridique du waqf dans la jurisprudence musulmane ; selon al-Ṭûsî (al-Mabsût), la définition est la suivante : taḥbîs al-aṣl wa-ițtâq al-man$f a^{\prime} a$, à savoir, un contrat qui entraîne l'inaliénabilité (tahbîs) de la nuepropriété d'un bien et la «libération » (ițlâq) de l'usufruit ${ }^{3}$.

En d'autres termes, il s'agit de rendre inaliénable un bien dont l'usufruit ou les revenus en provenant sont consacrés à un ou plusieurs bénéficiaires.

Le waqf est l'une des institutions socioculturelles religieuses majeures du monde musulman, en vigueur dans les sociétés musulmanes depuis les débuts de l'islam jusqu'à nos jours. Ce terme correspond dans la jurisprudence musulmane $(f i q h)$ à une donation faite à perpétuité par un particulier au bénéfice d'un autre individu, d'un ensemble de personnes ou d'une œuvre d'utilité publique, pieuse ou charitable.

Cependant, contrairement à une donation charitable (șadaqa), le waqf signifie que des revenus provenant du bien sont donnés en usufruit, le bien lui-même étant immédiatement inaliénable (habs) de manière permanente, ce qui donne à la donation son caractère de perpétuité. Si contrairement à d'autres formes de don et de charité comme l'aumône (zakât), le waqf est facultatif en islam, ou plus exactement « surérogatoire »; la pratique a été extrêmement répandue à différentes époques tout au long de l'histoire des civilisations musulmanes ${ }^{4}$.

\section{Le waqf dans la jurisprudence chiite}

Etant une pratique sociétale encadrée par la jurisprudence religieuse mais aussi par le droit étatique, les opérations du waqf sont définies par la jurisprudence musulmane quant à l'élaboration de ses conditions particulières et à

2 al-Ṭurayhî, 1375 / 1996, v. 4, p. 535 ; Ibn Manzûr Miṣrî, 1300 / 1883, lettre fấ, p. 395 ; Fathullâh, 1415 / 1995, Bâb al-wâw, p. 449.

3 Voir al-Ṭ̂̂sî, 1351 / 1973, v. 3, p. 286 ; Ibn Idrîs, 1411 / 199o, v. 3, p. 152 ; al-Muhaqqiq al-Hillî, 1409 / 1988, v. 2, p. 442 ; Fathullâh, 1415 / 1995, Bâb al-wâw, p. 449.

4 Neyestani, 2014, pp. 7-8. 
ses statuts. Du fait des divergences existant entre les différentes écoles (madhhabs) de l'islam, le waqf se présente également sous différentes formes qui, quoique assez similaires sur le fond, s'opposent parfois sur certains aspects non négligeables.

Dans le cas particulier du waqf, la jurisprudence chiite présente une caractéristique extrêmement importante : le terme waqf est lui-même présent dans les hadîths chiites ${ }^{5}$ et utilisé par les imams ${ }^{6}$. Cela trouve toute son importance dans le constat que cette pratique répandue à travers l'ensemble du monde musulman n'est citée sous cette désignation spécifique nulle part dans le Coran ni dans les hadîths du prophète Muhammad qui constituent les deux sources de la jurisprudence sunnite ${ }^{7}$. De ce fait, le waqf devient une interprétation des paroles du prophète, telles qu'elles peuvent être comprises dans certains hadîths. A l'opposé, les jurisconsultes (faqîhs) chiites s'appuient sur des hadîths supplémentaires pour asseoir la légitimité de la pratique, en particulier, ceux rapportés du sixième imam, Ja'far al-Șâdiq $(702-765 \mathrm{AD})^{8}$.

\section{Le waqf dans la Perse safavide}

Lorsque l'on s'intéresse à la notion de $w a q f$ dans l'histoire de la jurisprudence chiite, on est amené à considérer le rôle qu'elle joua durant les différentes périodes historiques, sachant que parallèlement au développement de la science de la jurisprudence et de son implantation dans la société, les jurisconsultes s'intéressèrent également à l'étude des instances sociales avec une précision toujours croissante. Dans cette recherche, nous nous intéresserons à l'ère géographique et historique qui correspond à l'Empire safavide, c'està-dire la Perse du $16^{\mathrm{e}}$ au $18^{\mathrm{e}}$ siècle car durant cette période, le waqf a connu une évolution radicale et fondamentale durant laquelle les chiites expérimentèrent l'utilisation de cette pratique à une large échelle au sein de la société. Pour la première fois, un gouvernement chiite indépendant vint au pouvoir en Iran avec une forme et un système de fonctionnement nouveau et les religieux prirent le contrôle de nombreuses fonctions civiles et culturelles.

A cette époque, la dynastie safavide avait créé un puissant état qui englobait l'Iran actuel, l'Irak actuel, le Khorasan et une partie de l'Afghanistan actuel. La jurisprudence devint l'une des composantes de la politique et du

5 Les paroles du prophète Muhammad et les 12 imams chiites.

6 Voir al-Ḥurr al-'Âmilî, 1403 / 1983, v. 13, pp. 292-332.

7 Neyestani, 2014, pp. 13-4.

8 Idem. 
gouvernement et les jurisconsultes religieux occupèrent des postes de grande responsabilité dans la politique et dans l'administration de l'Etat comme les postes de premier ministre (vizir), de responsable de religion (șadr, shaykh alislâm et mullâ bâshî), de juge ( $q$ âdi), etc. Les besoins de l'époque les amenèrent à développer les waqfs et tirer profit des capacités de cette institution. Cette période correspond à l'âge d'or du waqf d'un point de vue social dans la sphère religieuse chiite au point où certains rois safavides comme Shah 'Abbâs I établissent la totalité de leur richesse sous forme de waqf, entraînant, par leur exemple, un bon nombre des membres de leur cour dans cette pratique ${ }^{9}$.

\section{Les quatre piliers du waqf}

1) L'acte de waqf: la prononciation de la formule du waqf et l'acte du waqfen lui-même.

2) Le fondateur / la fondatrice (wâqif / wâqifa) du waqf, le donateur / la donatrice du bien mis sous le statut de waqf.

3) Le ou les bénéficiaire(s) du bien (mawqûf 'alayh). Il s'agit de la personne ou des personnes pouvant avoir la jouissance du bien ou de son usufruit en vertu de la désignation du fondateur du waqf.

4) Le bien mis en waqf par le fondateur (mawqûf).

Les deux piliers qui nous concernent dans cet article sont:

\section{Le fondateur / la fondatrice du waqf (wâqif / wâqifa)}

Le fondateur / la fondatrice du waqf constitue l'un des piliers du waqf et doit satisfaire à ses propres conditions. Les jurisconsultes ont extrapolé à partir des conditions propres aux contrats, partant du principe que le waqf est un contrat impliquant un transfert de propriété par le fondateur / la fondatrice du waqf.

Ainsi, le fondateur du waqf doit satisfaire aux mêmes conditions que pour les autres types de contrats (par exemple, la vente):

- être majeur (bâligh);

- être sain d'esprit et d'intention ('âqil);

- de ne pas être frappé d'incapacité spéciale (jâyiz al-tașarruf).

9 Ibid., p. 15 . 


\section{Le bien mis en waqf (mawqûf)}

Les jurisconsultes chiites s'accordent pour définir quatre conditions du bien mis sous waqf ${ }^{10}$ :

- il doit être physique ;

- il doit être une propriété licite du fondateur / de la fondatrice du waqf (cette condition montre que si le fondateur / la fondatrice n'est pas propriétaire de plein droit, il / elle n'a pas le droit de mettre le bien sous le waqf);

- sa prise en possession par le bénéficiaire doit être possible ;

- il doit survivre après l'utilisation de celui-ci.

Une des questions soulevées concernant le bien mis sous waqf est de savoir, puisqu'il est clairement établi comme condition du waqf que le fondateur / la fondatrice du waqf se dépouille entièrement de la propriété, s'il y a passation de la propriété à autrui ou non et qui, selon la jurisprudence chiite, devient le propriétaire du bien.

Dans le cas du waqf au bénéfice des particuliers: les bénéficiaires deviennent propriétaire du bien. À noter cependant que le bénéficiaire n'est pas propriétaire de plein droit, à savoir, il ne peut ni vendre ni céder ce waqf et ses héritiers n'en héritent pas s'ils n’ont pas été désignés par le fondateur / la fondatrice comme bénéficiaires : il est propriétaire dans le sens où il a la pleine et entière jouissance du bien.

Dans le cas du waqf au bénéfice de l'intérêt général : le bien devient propriété de Dieu.

Cette condition stipule qu'il est illicite pour le fondateur / la fondatrice d'être bénéficiaire de son waqf. Cette condition est attestée par des arguments scripturaires et par des arguments rationnels et fait l'unanimité absolue parmi les jurisconsultes chiites.

Voici les arguments avancés par les jurisconsultes concernés au sujet de cette condition :

- al-Karakî : «Et l'avis : (s'il fait waqf pour lui-même, c'est invalide) cela est parce qu'il est nécessaire pour que le bien soit mis sous waqf comme une sorte de propriété du fondateur / de la fondatrice alors il n'est pas rationnel que le waqf soit à son propre bénéfice ${ }^{11}$ ».

\footnotetext{
10 al-Baḥrânî, 1984, v. 22, pp. 176-81 ; al-Sabziwârî, 2002, v. 2, p. 6 ; al-Shahîd al-Thânî, 1993, v. 5, pp. 318-323 ; al-Karakî, 1987, v. 9, pp. 54-61.

11 al-Karakî, 1987, v. 9, p. 25 (traduit par moi-même).
} 
- al-Shahîd al-Thânî: «Il n'y a aucune controverse parmi nos Compagnons [les jurisconsultes chiites] au sujet de l'invalidité du waqf fait par une personne en son propre nom. Et cela parce que le waqf consiste en la perte de la propriété et le dépouillement du propriétaire en faveur du bénéficiaire. Dans ce cas, la propriété est déjà réalisée alors il n'y a pas de sens à rétablir ou renouveler [la propriété] avec l'établissement du waqf de même que de poser comme condition que le propriétaire profite de l'usufruit comme dans une vente ou une donation. Parce que le waqf est la passation de la propriété de l'usufruit seul ou de l'usufruit et de la propriété du bien à une autre personne et, donc, cela est illogique de passer la propriété à soi-même. Certains juristes sunnites ne sont pas d'accord à ce sujet et ont considéré cela comme valide en s'appuyant sur le fait que bénéficier d'un bien en vertu du waqf n'est pas la même chose que d'en bénéficier en vertu de la propriété et parfois l'intention est de mettre le bien en mainmorte et de s'interdire à lui-même de pouvoir passer la propriété de ce bien à un autre ${ }^{12}$ ».

\section{Le droit à la propriété aux femmes et son usage à l'époque safavide}

C'est par l'étude de plusieurs chartes de fondation de waqf se trouvant dans les archives d'Ispahan que nous examinons le droit de propriété tenu par les femmes dans cette société tel que nous pouvions le voir par le biais de cette documentation qui montre le lien entre la possession des femmes de la propriété foncière et bâtie et sa conversion en bien waqf. Autrement dit, les juristes musulmans (comme al-Karakîi ${ }^{13}$, al-Shahîd al-Thânî ${ }^{14}$, etc.) sont tous d'accord sur le fait qu'un individu, femme ou homme, doit posséder en pleine propriété (mulk) un bien avant de pouvoir le mettre en statut de bien waqf par le biais de l'établissement d'un waqf par sa charte de fondation (waqfiyya).

Les chartes du waqf créées par des femmes de l'époque safavide, notamment, à Ispahan du fait que notre documentation concerne cette ville, nous donnent des précisions concernant leurs œuvres caritatives et d'autres intérêts qu'elles avaient auprès de la société. Dans la jurisprudence chiite, le droit à la propriété de la femme est chose acquise mais dans la pratique, ceci ne correspondait pas toujours aux droits accordés par la jurisprudence chiite (l'acquisition de propriété par la voie d'héritage, de mariage, de la pratique de la dot mais aussi par l'achat ainsi que leur droit de disposer de cette propriété selon

\footnotetext{
12 al-Shahîd al-Thânî, 1993, v. 5, pp. 361-2 (traduit par moi-même).

13 al-Karakî, 1987, v. 9, p. 25.

14 al-Shahîd al-Thânî, 1993, v. 5, pp. 361-2.
} 
leur propres stratégies, tempérées tout de même par les pressions ou influences de leurs réseaux immédiats ou plus lointains). Ainsi, en théorie, elles peuvent effectuer des transactions avec les biens qu'elles possèdent sans avoir à obtenir la permission de quiconque et peuvent dépenser leur argent comme bon leur semble ou bien en faire don, y compris sous forme de waqf. Les chartes du waqf montrent que les femmes, particulièrement, celles de la famille royale ou de la haute société qui disposaient de richesses personnelles importantes ont réalisé des waqfs moyennant leurs biens à divers bénéficiaires.

\section{Exemples : genre, droit et propriété mise en waqf par des fondatrices safavides}

Parmi les chartes du waqf de l'Archive ${ }^{15}$ de l'Organisation des Waqfs et des CEuvres Caritatives d'Ispahan ${ }^{16}$ publiées dans le recueil, Documents des waqfs safavides, nous en étudions certaines qui remontent à l'époque safavide (du $16^{\mathrm{e}}$ au $18^{\mathrm{e}}$ siècle) et concernent des waqfs établis par des femmes d'Ispahan à cette époque.

Afin de s'approcher au plus près des détails concernant les biens possédés par les fondatrices safavides à partir de nos sources, nous analysons, à titre d'exemple, quelques chartes de ces waqfs.

Charte de $w a q f \mathrm{n}^{\circ} 106^{17}$

- Date : $1057 \mathrm{AH} / 1648 \mathrm{AD}$

- Fondatrice: Dilârâm khânum, épouse du Shah 'Abbâs I et arrière-grandmère du Shah 'Abbâs II ${ }^{18}$.

- Propriétés mises en waqf:

- les 19.5 parts de la totalité des 72 parts du village de Jalâdirân Quhâb dans la province d'Ispahan;

- les livres nommés dans la charte du waqf;

- la totalité des six dângs ${ }^{19}$ d'un hammâm (bain commun) dans le quartier de Nimâward à Ispahan ;

15 Cette archive se trouve dans le bâtiment de l'Organisation des Waqfs et CEuvres Caritatives d'Ispahan.

إيخانى سازمان اوقاف وامور خيرية اصفهان 16

17 Ashkiwarî, 1387 / 1430 / 2009, v. 7, pp. 198-213.

18 Aḥmadî, 2011, p. 87 .

19 Système de partition des biens immobiliers et terrains traditionnellement employé en Iran. Un dâng correspond à une part d'un sixième du bien, voir 'Amîd, 1389 / 2010, p. 512 ; Mu'în, 1992, harf dâl, v. 2, p. 1491. 
- la moitié des parts d'une résidence et trois boutiques situées au sud du bazar de Tchâhârsûq maqsûd ;

- 14 boutiques situées à côté de l'école Jadda kûchak.

- Bénéficiaire :

Pour l'entretien et les dépenses de l'école Jadda kûchak et ses résidents (les professeurs, les étudiants chiites duodécimains, le serviteur et le muezzin, à savoir, celui qui lance l'appel à la prière).

Charte de waqf $\mathrm{n}^{\circ} 103^{20}$

- Date : 1057 AH / 1648 AD

- Fondatrice : Hûnînâm khânum, mère du Shâh Safî et arrière-grand-mère du Shah 'Abbâs II ${ }^{21}$.

- Propriétés mises en waqf:

- des boutiques construites par la fondatrice situées à côté de l'école du village de Hurmuz Âbâd Ghuhâb, à savoir, une boutique de vente de tabac, un atelier de confiseries, une boulangerie, une boutique de porcelaines, un atelier de construction de fusils et deux boutiques situées dans l'école Jadda buzurg, bénéficiaire de ce waqf, dont un atelier de reliure et l'autre de dorure ;

- le village de Dahmard;

- le village de Barzâbâd Quhâb.

- Bénéficiaire :

Pour l'entretien de l'école Jadda buzurg et en cas de surplus, cela sera distribué aux étudiants résidant dans l'école si le gérant (mutawallî) le juge bon ainsi qu'aux enseignants et aux serviteurs.

Charte de waqf $\mathrm{n}^{\circ} 111^{22}$

- Date : $1071 \mathrm{AH} / 1661 \mathrm{AD}$

- Fondatrice : Guhar Shâh Biygum, fille de Mîrzâ Muhammad Ismấîl, épouse du Shaykh 'Âmir al-Najafî al-Jazâ'irî et mère de Shaykh Kalb 'Alî (elle n'appartient pas à la famille royale $)^{23}$.

- Propriétés mises en waqf:

- la totalité des six dângs de la ferme connue et désignée sous le nom de «la ferme Jiân » située dans la circonscription de Quhâb, l'une des neuf circonscriptions d'Ispahan ;

\footnotetext{
$20 \quad$ Ashkiwarî, 1387 / 1430 / 2009, v. 5, pp. 398-437.

21 Aḥmadî, 2011, p. 88.

22 Ashkiwarî, 1387 / 1430 / 2009, v. 7, pp. 214-45.

23 Aḥmadî, 2011, p. 202.
} 
- la part correspondant à quatre dângs et demi de la ferme connue et désignée sous le nom de «ferme d'Anduwân» située dans la même circonscription.

- Bénéficiaire :

La moitié des revenus est au bénéfice des pèlerins et des résidents du sanctuaire de l'imam 'Alî à Nadjaf et l'autre moitié est au bénéfice des pèlerins et des résidents du sanctuaire de l'imam Ḥusayn à Karbala.

Charte de $w a q f \mathrm{n}^{\circ} 15 \mathrm{o}^{24}$

- Date : $1079 \mathrm{AH} / 1668 \mathrm{AD}$

- Fondatrice : la mère du Shâh Sulaymân safavide.

- Propriété mise en waqf:

- Les dix fermes à Dâr imârây 'ulyâ wa-suflâ à Karwan dans la province d'Ispahan.

- Bénéficiaire :

Pour l'éclairage et les dépenses des sanctuaires de l'imam 'Alî (à Nadjaf), de l'imam Ḥusayn (à Karbala), de l'imam Kâzim (à Bagdad), de l'imam Jawâd (à Bagdad), de l'imam Hâdî (à Samarra), de l'imam Ḥasan al-'Askarî (à Samarra) et pour les pauvres et les résidents de ces lieux saints.

Charte de $w a q f \mathrm{n}^{\circ} 5^{25}$

- Date $: 1083 / 1672$

- Fondatrice : Dahdah Khâtûn (elle n'appartient pas à la famille royale $)^{26}$.

- Propriétés mises en waqf:

- une résidence avec des chambres, située dans le quartier de Nimâward à Ispahan ;

- toutes les boutiques qui sont à côté de cette résidence.

- Bénéficiaire:

Waqf au bénéfice de sa fille Razîyyah bânû Khânum et après elle (après son décès), en faveur de l'ensemble de ses descendants, filles ou garçons, à titre héréditaire en ce qui concerne les parts de revenus qu'ils reçoivent. Ensuite, au cas où la ligne vient à s'éteindre, les revenus seraient pour les nécessiteux chiites duodécimains.

Charte de $w a q f \mathrm{n}^{\circ} 27^{27}$

- Date : $1090 / 1679$

\footnotetext{
24 Ashkiwarî, 1387 / 1430 / 2009, v. 7, pp. 291-331.

25 Ibid., v. 2, pp. 389-97.

26 Ahmadî, 2011, p. 176.

27 Ashkiwarî, 1387 / 1430 / 2009, v. 5, pp. 228-40.
} 
Fondatrice : Murwârîd Khânum, fille d'Âga Ḥusayn (elle n'appartient pas à la famille royale $)^{28}$.

- Propriété mise en waqf:

- les cinq maisons situées dans le quartier de Sanji à Ispahan.

- Bénéficiaire :

Pour l'éclairage et parfumer les lieux saints des 14 infaillibles (le prophète de l'islam, sa fille Fâțima et les 12 imams chiites).

Charte de $w a q f \mathrm{n}^{\circ} 6^{29}$

- Date: 1092 / 1681

- Fondatrice : Zubaydih Biygum, fille du Shâh Sulaymân safavide ${ }^{30}$.

- Propriétés mises en waqf:

- la totalité des parts du village de Bardchâ' dans la province d'Ispahan;

- les 15 parts de la totalité des 72 parts du village de Khyrâbâd dans la province d'Ispahan ;

- la totalité du village de Châdân et la ferme de Tchâhak dans la province d'Ispahan ;

- les 12 parts de la totalité des 72 parts de la ferme de Tchah linjân dans la province d'Ispahan;

- les 6 parts de la totalité des 72 parts de la ferme de Tâd linjân dans la province d'Ispahan ;

- la totalité des parts de la ferme de Haydar Âbâd et la ferme de Zunh dans la province d'Ispahan ;

- la totalité des parts de la ferme de Ḥasan Âbâd dans la province d'Ispahan;

- la totalité des parts d'un jardin dans le village d'Ilyâdirân Mârbîn dans la province d'Ispahan;

- la totalité des parts de la ferme de Khâvih dans la province de Qum;

- la totalité des parts de la ferme de Mazbûrah dans la province de Qum ;

- les 21 boutiques qui sont désignées connues à Ispahan.

- Bénéficiaire :

Pour le sanctuaire de l'imam Ḥusayn à Karbala et pour les cérémonies de deuil de 'Âshûrâ (le jour du martyre de l'imam Ḥusayn, $3^{\mathrm{e}}$ imam du chiisme)

Charte de $w a q f \mathrm{n}^{\circ} 8^{31}$

- Date : $1101 / 1690$

\footnotetext{
28 Aḥmadî, 2011, p. 176.

29 Ashkiwarî, 1387 / 1430 / 2009, v. 1, pp. 32-52.

30 Aḥmadî, 2011, p. 201.

31 Ashkiwarî, 1387 / 1430 / 2009, v. 1, pp. 70-87.
} 
- Fondatrice : Badri Jahân Khânum, fille de Mîrzâ Muhammad Mas'ûd, ancien vizir (ministre) de la ville de Mâzandarân ${ }^{32}$.

- Propriétés mises en waqf:

- une résidence en deux étages et un caravansérail situés derrière l'école Jadda kûchak ;

- les 5 boutiques à côté de l'école Jadda kûchak ;

- la moitié des parts d'une résidence et deux boutiques situées au sud de bazar de Tchâhârsûq maqsûd.

- Bénéficiaire :

Les revenus du waqf sont au bénéfice du fils de la fondatrice, Mîrzâ 'Ulyâ, pendant le vivant de la fondatrice et après sa mort, les revenus sont pour Mîrzâ 'Ulyâ et les autres enfants de la fondatrice à parts égales pour les filles et les garçons et aussi pour la petite fille de la fondatrice nommée Charaf Jahân khânum dont la mère est décédée lors de la création du waqf, puis après eux, pour l'ensemble de ses descendants, filles ou garçons, à titre héréditaire.

Ensuite, au cas où la ligne vient à s'éteindre, les revenus seraient pour les étudiants chiites en science religieuse résidants à Ispahan.

Charte de $w a q f \mathrm{n}^{\circ} 5^{21^{33}}$

- Date : 1103 / 1692

- Fondatrice: Zaynab Biygum, fille d'Amîr Muhammad Ardistânî (elle n'appartient pas à la famille royale.) ${ }^{34}$

- Propriétés mises en waqf:

- tous les 11 jardins situés à Ardistân dans la province d'Ispahan.

- toutes les 20 terres cultivées à Ardistân dans la province d'Ispahan.

- Bénéficiaire :

Pour les nécessiteux ( 2 tûmâns ${ }^{35}$ par personne). La fondatrice n’a pas précisé dans la charte du waqf s'il s'agissait des nécessiteux d'Ardistân ou d'ailleurs.

Charte de waqf $\mathrm{n}^{\circ} 105^{36}$

- Date : $1113 / 1702$

\footnotetext{
32 Aḥmadî, 2011, p. 180.

33 Ashkiwarî, 1387 / 1430 / 2009, v. 8, pp. 340-73.

34 Aḥmadî, 2011, p. 176.

35 Unité monétaire.

36 Ashkiwarî, 1387 / 1430 / 2009, v. 6, pp. 6-63.
} 
- Fondatrice: Ziynab Biygum, épouse de Hakîm al-Malik (elle n’appartient pas à la famille royale. $)^{37}$.

- Propriétés mises en waqf:

- les 3.5 parts de la totalité des 72 parts du village de Hawâkân Mârbîn dans la province d'Ispahan ;

- un vignoble dans le village de Mârân Mârbîn dans la province d'Ispahan ;

- un caravansérail à côté de l'école ;

- les 8 boutiques à côté de l'école ;

- une maison à côté de l'école ;

- une terre connue dans le village de Mârân Mârbîn dans la province d'Ispahan.

- Bénéficiaire :

Pour l'entretien de l'école Nîmâward créée par la fondatrice en 1113 / 1702 située dans le bazar d'Ispahan, et pour les étudiants chiites résidants dans cette école.

Charte de $w a q f \mathrm{n}^{\circ} 40^{38}$

- Date : $1131 / 1719$

- Fondatrice: Hiishmat al-nisâ' khânum, fille de Mîrzâ Muhạammad Rị̣â Munajjim Bâshî (elle n’appartient pas à la famille royale.) ${ }^{39}$

- Propriétés mises en waqf:

- les 2 parts de la totalité des 72 parts du village d'Afjid al-najân dans la province d'Ispahan ;

- les 4 boutiques dans le quartier de Nîmâward à Ispahan.

- Bénéficiaires :

- 3 tûmâns annuel pour la récitation du Coran ;

- 2 tûmâns annuel pour Parî rukhsâr (l'une des servantes de la fondatrice);

- 1 tûmân annuel pour chacun des autres servantes da la fondatrice;

- le reste à la discrétion du gérant (mutawallî) pour les bonnes œuvres ;

- après le décès des servantes, leurs parts seraient dépensées aux bonnes œuvres.

37 Aḥmadî, 2011, p. 90.

38 Ashkiwarî, 1387 / 1430 / 2009, v. 7, pp. 73-86.

39 Aḥmadî, 2011, p. 177. 


\section{Le rôle et l'importance des waqfs pour les écoles (madrasas) à l'époque safavide}

Durant la période safavide en Iran, la construction d'écoles (madrasas) et de centres d'enseignement connut un essor important non seulement dans la capitale d'Ispahan mais aussi dans de nombreuses villes du royaume, grandes comme petites. Cette prolifération d'écoles religieuses transforma la Perse safavide en l'un des principaux centres d'apprentissages de sciences religieuses du chiisme: position qu'elle gardera jusqu'à la chute d'Ispahan et la fin de la dynastie safavide. Mise à part la politique de propagande intensive du gouvernement pour développer le chiisme duodécimain en Iran, le waqf joua un rôle non négligeable dans ce processus de développement des centres d'enseignement.

Toutes les écoles construites durant la période safavide furent financées soit par le gouvernement soit par des waqfs ce qui, en soi, en dit long sur l'importance des waqfs consacrés à la fondation de centres d'enseignement d'autant que certains rois safavides eux-mêmes consacrèrent des sommes importantes de leur fortune personnelle à la construction d'écoles ${ }^{40}$.

Les waqfs destinées à subventionner des établissements d'enseignement religieux ne se limitaient pas à la construction des bâtiments eux-mêmes puisque les fondateurs / fondatrices des waqfs prévoyaient également des revenus destinés à l'entretien des lieux et aux réparations éventuelles mais aussi pour assurer la subsistance des étudiants. Souvent les donateurs / donatrices qui n'avaient pas les moyens de financer la construction et l'entretien d'une école toute entière créaient des waqfs au bénéfice d'une école existante.

Il ne faut pas oublier que la fondation d'une école n'était pas une œuvre pieuse à la portée de tout le monde et seules les personnes plus riches pouvaient se le permettre. La pérennité d'une école dépendait aussi, en grande partie, de sa réputation et de son rayonnement qui, à leur tour, dépendaient de la qualité des enseignants et des étudiants. Tout ceci nécessitait bien évidemment des dépenses importantes. De ce fait, les waqfs étaient également établis pour satisfaire les besoins des enseignants et des élèves, payer des salaires aux premiers et des aides aux dépenses quotidiennes aux seconds, ceci afin d'assurer la pérennité de l'établissement ${ }^{41}$.

Rappelons, pour finir, que les waqfs à destination des écoles ont été, durant toutes les époques musulmanes parmi les waqfs les plus importants car outre le coût de construction de l'école, des waqfs devaient être établis pour en assurer le fonctionnement sur la durée.

$\begin{array}{ll}40 & \text { Ibid., pp. 43-5. } \\ 41 & \text { Ibid., pp. 46-7. }\end{array}$ 
L'un des facteurs qui influençait directement et indirectement le développement qualitatif et quantitatif des écoles en Perse était lié à la politique religieuse de la dynastie des Safavides qui établissaient les bases de leur autorité et de leur légitimité sur le socle de l'islam chiite dès la création de leur royaume. Dans ces conditions, il était naturel pour que le renforcement des fondations joue un rôle politique et que la pérennité de la dynastie passe par la création d'une assise populaire solide s'appuyant sur la foi. Cela, d'autant plus qu'avec l'officialisation de l'islam chiite duodécimain en tant que religion de l'Empire safavide, des tensions apparurent avec les royaumes voisins qui étaient tous d'obédience sunnite et sans oublier qu'à l'arrivée au pouvoir des Safavides, le chiisme n'était pas la religion majoritaire en territoire persan ${ }^{42}$.

De ce fait, la propagation et l'implantation profonde de la nouvelle religion officielle en Iran demandaient des mesures urgentes et radicales. La première mesure fut d'inviter des savants religieux de Jabal 'Âmil (dans le sud du Liban actuel) et d'autres régions pour assurer les conditions idéales afin de réunir des penseurs, jurisconsultes et autres savants chiites dans la capitale du nouvel empire safavid. Une fois cette étape réalisée, il devenait nécessaire de fournir à ces personnes les moyens de procéder efficacement à l'enseignement et à la propagation des préceptes de la foi. Ainsi, les écoles religieuses commencèrent à se développer. Ce mouvement, une fois amorcé, ne s'arrêta qu'à la fin de l'époque safavide : toujours plus d'écoles religieuses ouvrant leurs portes à travers le royaume sans se limiter à la capitale d'Ispahan mais aussi à d'autres centres religieux comme Machhad et Qum³ ${ }^{43}$

Les exemples mentionnés ci-dessus nous permettent de classer les waqfs établis par les femmes d'Ispahan dans les catégories suivantes:

1. waqfs pour l'entretien, l'éclairage et les dépenses des écoles religieuses (Jadda buzurg, Jadda kûchak, Nîmâward);

2. waqfs pour les descendants et descendantes des fondatrices;

3. waqfs pour l'entretien, l'éclairage et parfumer des lieux saints (comme le mausolée du prophète de l'islam ou des imams chiites);

4. waqfs pour les servantes, les nécessiteux, les pèlerins et les résidents des lieux saints ou pour les bonnes œuvres.

Nous constatons ainsi que l'un des objets des waqfs fondés par des femmes de la période safavide dans nos exemples étaient les écoles religieuses. Ainsi, les femmes jouèrent un rôle non négligeable dans l'édification de très nombreuses

\footnotetext{
$42 \quad$ Neyestani, 2014, p. 271.

43 Idem.
} 
écoles qui fleurirent à Ispahan après que cette ville devint la capitale de la dynastie chiite des Safavides.

Les journaux de voyage des Européens en Iran à l'époque safavide sont parmi les sources historiques les plus importants pour étudier cette période car ils mettent à disposition des informations précises sur cette époque qui ne se trouvent pas toujours dans d'autres sources historiques.

Jean Chardin (1643-1713) est l'un des voyageurs européens qui a séjourné en Iran à l'époque safavide et a visité Ispahan ${ }^{44}$ en $1665^{-1670}$ et ensuite en 16711677. Il évalue le nombre d'écoles d'Ispahan à $57^{45}$ durant l'époque safavide. Or seules les chartes du waqfs de 17 madrasas nous sont parvenues. Ces chartes sont conservées dans l'Archive de l'Organisation des Waqfs et des OEuvres Caritatives d'Ispahan.

Ci-dessus, parmi nos exemples, nous avions mentionnés trois de ces waqfs en faveur des écoles établies par des femmes. Dans l'ordre chronologique de leur création, ces écoles sont :

- l'école Jadda kûchak, fondée en 1056 / 1647 par Ḥûrînâm khânum, mère du Shâh Safî et arrière-grand-mère du Shah 'Abbâs II ${ }^{46}$.

- l'école Jadda buzurg, fondée en 1057 / 1648 par Dilârâm khânum, épouse du Shah 'Abbâs I et arrière-grand-mère du Shah 'Abbas II ${ }^{47}$.

- l'école Nîmâward, fondée en 1113 / 1702 par Ziynab Biygum, épouse de Ḥakîm al-Malik Ardistânîn ${ }^{48}$.

Comme nous l'avions remarqué, la construction d'une école peut représenter un montant très important, ce qui illustre que le niveau socio-économique important des fondatrices. Sur ces trois fondatrices d'école, les deux premières (Dilârâm khânum et Ḥûrînâm khânum) étaient de proches parents du souverain et membres de la famille royale. Seule la troisième, Zaynab Biygum, épouse de Hakîm al-Malik fait exception car elle était l'épouse d'un médecin ${ }^{49}$. Ces fondatrices avaient prévu des waqfs dont les revenus seraient destinés à assurer l'entretien du bâtiment de l'école, les dépenses courantes et les salaires des employés de l'école ainsi que les dépenses des étudiants.

\footnotetext{
44 Chardin, 1993, v. 1, pp. 11-2.

45 Chardin, 1711, v. 5, p. 25.

46 Aḥmadî, 2011, p. 88.

47 Ibid., p. 87 .

48 Ibid., p. 90.

49 Ibid., pp. 43-5.
} 


\section{Conclusion}

Les 230 ans de règne des Safavides en Iran ont provoqué une métamorphose radicale de l'ensemble de la société et marqua le pays d'un sceau indélébile. En effet, depuis la première islamisation de la région à l'issue de la conquête arabe majoritairement sunnite et malgré plusieurs tentatives infructueuses à différentes époques pour répandre plus largement la doctrine chiite imâmite, la Perse était largement sunnite. Malgré la présence d'une minorité imamite et de sympathisants de leur cause, créant ainsi l'apparition sporadique de petits royaumes chiites sur le territoire, la population restait, dans son ensemble, ancrée dans un sunnisme classique qui l'intégrait, du moins sur le plan strictement religieux, aux autres grandes entités de la région.

Or dès leur arrivée au pouvoir, les Safavides s'attelèrent à propager par tous les moyens le chiisme duodécimain et de l'asseoir comme religion et doctrine officielles de leur empire. Cela malgré les réticences qui s'étendaient jusqu'à leurs partisans de la première heure, peu convaincus de la nécessité de s'attirer l'animosité de la majorité du peuple. Contre toute attente, les Safavides réussirent là où d'autres, avant eux, avaient échoué en réalisant une métamorphose de la société sur le plan religieux et culturel, métamorphose qui ne se trahit pas avec l'effondrement de l'Empire safavide contrairement à ce qui avait été le cas, par exemple, du califat Fatimide d'Egypte. Autrement dit, les Safavides étaient parvenus à faire de la Perse une terre chiite.

Comme évoqué ci-dessus, la pratique du waqf rendit cela possible tout en présentant des avantages non négligeables par rapport à une prise en charge directe par le gouvernement des instances dans la société. De nombreux biens furent mis en waqf, les souverains safavides sachant tirer profit de cette pratique dans l'objectif de renforcer leur pouvoir à travers l'expansion de la foi chiite.

De ce fait, la quantité, la richesse et la compétence du niveau académique des écoles dans l'Empire safavide augmentaient en parallèle de leur influence sur la vie culturelle et religieuse dans les territoires safavides. Le waqf ne concernait d'ailleurs pas exclusivement les écoles puisque nombre d'entre eux étaient destinés à des buts pieux ou religieux que ce soit pour l'entretien des lieux saints chiites ou des besoins de pèlerins desdits lieux saints ou, enfin, pour la réalisation de cérémonies religieuses comme la commémoration de 'Âshûrâ. Nous constatons encore une fois le fait que l'usage du waqf safavide œuvrait dans le sens d'implanter et de renforcer la propagation du chiisme en tant que nouvelle religion officielle de l'Empire.

Si le waqf joua un rôle important dans l'élaboration de l'identité de la société safavide, c'est aussi parce que les jurisconsultes chiites surent adapter 
cette pratique aux besoins de la religion imamite et surtout l'organiser en promulguant des statuts propres au waqf chiite, permettant ainsi aux souverains d'utiliser cette pratique dans le sens de leurs objectifs religieux et politiques. Une synthèse des textes des chartes du waqf disponibles dans l'Archive de l'Organisation des Waqfs et des CEuvres Caritatives d'Ispahan fait apparaitre des bénéficiaires qui, aux yeux d'un jurisconsulte sunnite, seraient totalement incongrus, voire inadmissibles.

En réalité, la proportion des waqfs établis au bénéfice des descendants du fondateur ou pour une cause en particulier s'apparentant à une bonne œuvre ou à une cause d'intérêt général comme cela a été le cas pour les premiers waqfs, selon ce que l'on peut trouver dans les hadîths, est loin d'être dominante dans notre échantillon. Au contraire, il semble que la notion de la «bonne action » soit devenue de plus en plus synonyme d'action en faveur de l'implantation du chiisme, que ce soit à travers le soutien aux écoles religieuses, aux savants chiites ou aux cérémonies rituelles exclusivement chiites. Pour que de tels waqfs deviennent possibles, il aurait nécessairement fallu que des jurisconsultes valident et justifient par la pratique théorique de l'ijtihâd la licéité de tels bénéficiaires. Le waqf deviendra ainsi l'outil de choix pour l'implantation de la foi mais aussi pour améliorer de manière sensible les conditions socioculturelles de l'époque à travers la création d'hôpitaux et d'orphelinats mais aussi de systèmes d'irrigation et de ponts comme cela a été le cas ailleurs en terre d'islam, chiite et sunnite.

Ainsi, en s'appuyant sur plusieurs exemples donnés ici, on constate que les femmes dans la société safavide et, par ailleurs, dans la société musulmane dans son ensemble, jouissaient de plein droit de leurs biens car sans avoir accès à la propriété de plein droit, les fondatrices ne pouvaient pas en théorie créer des waqfs. En effet, nos documents précisent des situations où les femmes créaient des fondations waqf en tant qu'actes charitables, montrant qu'elles participaient activement dans la construction de leur société, jouissant du fait que la jurisprudence musulmane leur accordait le droit à posséder la pleine propriété en leur nom mais aussi avec une possibilité d'en disposer à leur gré.

Tout cela nous permet de conclure que la question du genre et des droits de propriété ne posait guère de problème quant à la création d'un waqf. Les documents montrent que les fondatrices de waqf dans la capitale safavide s'intéressaient aux questions culturelles, religieuses et sociales de la société dans laquelle elles vivaient et qu'elles s'y investissaient, dans la mesure de leurs moyens et grâce à leur droit de propriété, en consacrant une partie de leurs biens aux œuvres d'intérêt général et caritatives à travers le waqf. 


\section{La transcription scientifique des caractères arabes et persans}

Dans cette recherche, afin de rendre plus lisible des mots en arabe et en persan à tous ceux qui connaissent la langue arabe classique ou le persan ainsi qu'à tous ceux qui souhaitent connaître l'orthographe exacte des termes et des noms utilisés, nous avons adopté le système ci-dessous de translittération.

\begin{tabular}{|c|c|c|}
\hline أ & a & ص \\
\hline ! & $\mathrm{i}$ & ض \\
\hline 1 & $\mathrm{u}$ & $b$ \\
\hline 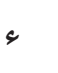 & , & ظ \\
\hline ب & $\mathrm{b}$ & $\varepsilon$ \\
\hline ب & $\mathrm{p}$ & $\dot{\varepsilon}$ \\
\hline ت & $\mathrm{t}$ & ف \\
\hline ث & th & ق \\
\hline ج & $\mathrm{j}$ & 5 \\
\hline ॄ & ch & 3 \\
\hline$\tau$ & ḥ & $J$ \\
\hline$\dot{\tau}$ & $\mathrm{kh}$ & دـ \\
\hline د & $\mathrm{d}$ & $\dot{ن}$ \\
\hline j & $\mathrm{dh}$ & $ه$ \\
\hline J & $\mathrm{r}$ & 9 \\
\hline j & $\mathrm{z}$ & ي \\
\hline j & $\mathrm{zh}$ & $\tau$ \\
\hline س & $\mathrm{S}$ & اي \\
\hline ش & $\mathrm{sh}$ & او \\
\hline
\end{tabular}




\section{Bio}

Mohammadreza Neyestani is a researcher in Islamic Studies and modern history affiliated with TELEMME (MMSH), Aix-Marseille University and teaches in the Department of History at Isfahan University, Iran where his courses concern Shiite history and jurisprudence within an interdisciplinary perspective from the viewpoint of Islamic religious science. He obtained a master's in Islamic Studies and modern Muslim civilization at the EPHE (Ecole Pratique des Hautes Etude) Paris-Sorbonne and received a PhD in Middle Eastern modern history (Mondes arabe, musulman et hamito-sémitique) from AixMarseille University. He works on the waqf pious foundations created during the Safavid period in Iran within the Twelver Shiite tradition.

\section{Bibliographie}

\section{Archives}

Ârchîwî Sâzmâni awqâf wa umûri khiyrîyyihi Isfahân, Archive de l'Organisation des Waqfs et des CEuvres Caritatives d'Ispahan, Ispahan, Iran

\section{Liste des chartes du waqf utilisées}

Charte de waqf $\mathrm{n}^{\circ} 6$

Charte de waqf $\mathrm{n}^{\circ} 8$

Charte de waqf $\mathrm{n}^{\circ} 27$

Charte de waqf $\mathrm{n}^{\circ} 40$

Charte de waqf $\mathrm{n}^{\circ} 5^{\circ}$

Charte de waqf $\mathrm{n}^{\circ} 103$

Charte de waqf $\mathrm{n}^{\circ} 105$

Charte de waqf $\mathrm{n}^{\circ} 106$

Charte de waqf $\mathrm{n}^{\circ} 111$

Charte de waqf $\mathrm{n}^{\circ} 150$

Charte de waqf $\mathrm{n}^{\circ} 5^{21}$ 


\section{Publications}

'Allâma (al-) al-Ḥillî, al-Ḥasan b. Yûsuf, 1410 AH / 1990 AD, al-Irshâd al-adhhân, 1 ere éd., 2 v., Qum, Mu'assasat al-nashr al-islâmî.

'Amîd, Ḥasan, 1389 solaire / 2010 AD, Farhang fârsîjî̀bî 'Amîd (Dictionnaire persan 'Amîd) Téhéran, Râhi Rushd.

Aḥmadî, Nuzhat, 2011, «Dar bâbi awqâfî șafawî (majmûiyi maqâlât)» (Concernant les

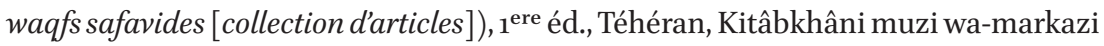
asnâdi majlisi shurâyi islâmî (Bibliothèque et Centre d'archive de l'Assemblée Nationale).

Ashkiwarî, Sâdiq, 1387 solaire / 1430 AH / 2009 AD, Asnâdi mawqûfât Ispahan (Documents des waqfs d'Isphan), 1 ere éd., sous contrôle de l'Organisation des Waqfs et des CEuvres Caritatives d'Ispahan, 12 v., Qum, Majma'i dhakhâ'ri islâmî.

Baḥrânî (al-), Yûsuf, 1984, Hadâ'iq al-nâdira fî ahkâm al-itra al- țâhira, (Les jardins resplendissants à l'égard des dispositions données par les proches [du prophète]), Qum, Mu'assasat al-nashr al-islâmî.

Calmard, Jean, 1993, Etudes safavides, Paris, Éditions d'IfRI (Institut Français de Recherche en Iran).

Chardin, Jean, 1711, «Voyages de Monsieur le chevalier Chardin en Perse et autres lieux de l'Orient», $B N F$, Édité par J.-L. de Lorme (Amsterdam), disponible en ligne sur http://gallica.bnf.fr/ark:/12148/bpt6k1050392t.r=Jean+Chardin+\%3A+Voyage+du+ chevalier+Chardin+en+perse+et+autre+lieu+de+l\%27orient.langEN, date d'accès : 14 mars 2017.

Chardin, Jean, 1993, «Voyages de Monsieur le chevalier Chardin en Perse et autres lieux de l'Orient », traduit par Iqbâ Yagmâyî, (Safarnâmih Shuwâliyih Chardin), Téhéran, Tûs.

Deguilhem, Randi, 2003, «Gender Blindness and Societal Influence in Late Ottoman Damascus: Women as the Creators and Managers of Endowments », HAWWA: Journal of Women of the Middle East and the Islamic World, Leiden, Brill, $\mathrm{n}^{\circ} 1 / 3$, pp. 329-50.

Fatḥullâh, Aḥmad, 1415 AH / 1995 AD, Mújam alfâz fiqh al-Jaffarî (Collection des expres-

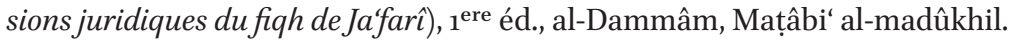

Ḥurr (al-) al-'Âmilî, Muhammad b. Ḥasan, 1403 AH / 1983 AD, al-Wasấil al-shîa, (Les moyens du chiisme), 20 v., Beyrouth, Dâr ihyâ' al-turâth al-'arabî.

Ibn Idrîs, Muhammad b. Aḥmad al-Ḥillî, 1411 AH / 1990 AD, al-Sarẩir, (Les secrets), $2^{\mathrm{e}}$ éd., Qum, Mu'assasat al-nashr al-islâmî.

Ibn Manẓûr Mișrî, Muhammad b. Mukram, 1300 AH / 1883 AD, Lisân al-'arab (La langue

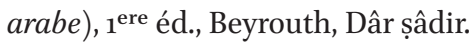


Karakî (al-), Nûr al-dîn, 1987, Jâmi al-maqâșid fî sharh al-qawâ'id, (Remplissant tous les objectifs pour expliquer les règles), Qum, Mu'assasat Âl al-bayt li-ihyyâ' al-turâth.

Lambton, Ann K. S., 2005, «Waḳf en Perse », Encyclopédie de l'Islam, édité par Th. Bianquis, C. E. Bosworth, E. van Donzel, W. P. Heinrichs et P. J. Bearman, v. XI, Leiden, Brill, pp. 89-95.

Mu'în, Muḥammad, 1992, Farhang fârsî Mu'în (Dictionnaire persan Mu'în), 8e éd., 6 v., Téhéran, Amîr kabîr.

Muḥaqqiq (al-) al-Ḥillî, Ja'far b. Ḥasan, 1409 AH / 1988 AD, Sharâyi‘ al-islâm, (Les lois de l'islam), 2 éd., Istiqlâl.

Neyestani, Mohammdreza, 2014, Fondations waqf dans le chiisme duodécimain en Iran du $16^{\text {ème }}$ au $18^{\text {ème }}$ siècle selon les hadîths, le fiqh et la société safavide, thèse de doctorat, sous la direction de Randi Deguilhem, Aix-Marseille Université (AMU), Aix-en-Provence.

Peters, R., 2005, «Waḳf selon le droit musulman classique», Encyclopédie de l'Islam, édité par Th. Bianquis, C. E. Bosworth, E. van Donzel, W. P. Heinrichs et P. J. Bearman, v. XI, Leiden, Brill, pp. 65-70.

Sabziwârî (al-), Muḥammad Bâqir, 2002, Kifâyat al-ạ̣kâm, Suffisant pour les prescriptions), Qum, Mu'assasat al-nashr al-islâmî.

Savory, Roger Mervyn, 1980, Iran under the Safavids, Presses Universitaires Cambridge, traduit en persan par Kâmbîz 'Azîzî, 2006, Irâni 'aṣri Șafavî, Téhéran, Markaz.

Shahîd (al-) al-Thânî, Zayn al-dîn al-'Âmilî, 1993, Masâlik al-afhâm fi tanqîh Sharâyi' alislam (Chemins des compréhensions pour la révision du livre Sharâyi' al-islâm), Qum, Mu'assasat al-maârif al-islâmiyya.

Ṭurayhî (al-), Fakhr al-dîn, 1375 solaire / 1996 AD, Majma' al-Bahrayn (Confluent des deux mers), 3 éd., annoté par A. Husaynî, Téhéran, al-Maktabat al-Murtaḍawîya.

Ṭûsî (al-), Muhammad b. Hasan, 1351 solaire / 1973 AD, al-Mabsûț (Ce qui est expliqué) annoté par M. B. al-Bihbûdî, 8 v., Téhéran, al-Maktabat al-Murtaḍawîya. 